\title{
Correlation between Automated Quantification of Lung Perfusion Blood Volume (PBV) and Pulmonary Artery Pressure
}

\author{
Hirofumi Koike1, Eijun Sueyoshi1 ${ }^{*}$, Ichiro Sakamoto ${ }^{1}$, Akira Tsuneto², \\ Masataka Uetani ${ }^{1}$ \\ ${ }^{1}$ Department of Radiology, Nagasaki University School of Medicine, Nagasaki, Japan \\ ${ }^{2}$ Department of Cardiovascular Medicine, Nagasaki University Hospital, Nagasaki, Japan \\ Email: *sueyo@nagasaki-u.ac.jp
}

Received 23 March 2016; accepted 4 June 2016; published 7 June 2016

Copyright (C) 2016 by authors and Scientific Research Publishing Inc.

This work is licensed under the Creative Commons Attribution International License (CC BY). http://creativecommons.org/licenses/by/4.0/

(c) (i) Open Access

\section{Abstract}

Purpose: Dual-energy CT (DECT) can be used for quantification of lung perfusion blood volume $(\mathrm{PBV})$, allowing objective evaluation. However, no reports have investigated pulmonary perfusion correlating with pulmonary artery pressure (PAP) in patients with chronic pulmonary diseases. The purpose was to evaluate automated quantification of the lung PBV using dual-energy CT, and its correlation with PAP. Methods: 274 patients who underwent echocardiography within two weeks also underwent $C T$. The population was divided into high $(\geq 40 \mathrm{mmHg})$ and low $(<40 \mathrm{mmHg})$ estimated systolic PAP (sPAP) groups ( $n=63$ and $n=211$, respectively). We retrospectively evaluated the lung PBV using Syngo software, and correlations between the lung PBV and estimated sPAP. Results: Lung PBV values were $25.0 \pm 9.6$ and $29.0 \pm 9.3$ Hounsfield units (HU) in high and low SPAP groups, respectively, with a significant difference between them $(p=0.003)$. In the high sPAP group with underlying lung diseases $(n=15)$, chronic thromboembolism $(n=25)$, pulmonary artery stenosis $(n=12)$, and left heart failure $(n=11)$, using the Dana Point classification system, lung PBV values were $18.6 \pm 1.6,25.1 \pm 4.5,25.8 \pm 4.5$, and $32.7 \pm 9.4 \mathrm{HU}$, respectively. There were significant differences in quantification of the lung PBV among them. The mean sPAP of subjects with left heart failure was significantly higher than in the others. In subjects with left heart failure, a positive correlation between the lung PBV value and SPAP was noted $(R=0.721, p$ $<0.0001)$. Conclusions: Automated quantification of the lung PBV may estimate the high sPAP. The lung PBV may contribute to clarifying the etiology of a high PAP due to left heart failure.

"Corresponding author.

How to cite this paper: Koike, H., Sueyoshi, E., Sakamoto, I., Tsuneto, A. and Uetani, M. (2016) Correlation between Automated Quantification of Lung Perfusion Blood Volume (PBV) and Pulmonary Artery Pressure. Open Journal of Radiology, 6, 105-112. http://dx.doi.org/10.4236/ojrad.2016.62016 
Keywords

Dual-Energy CT, Lung Perfusion Blood Volume, Pulmonary Hypertension, Iodine Attenuation

\section{Introduction}

Recently, dual-energy CT systems have been widely used for the various organs. Dual-energy CT pulmonary angiography does not expose subjects to any significant additional radiation beyond that of standard CT pulmonary angiography [1]-[4]. Post-processing software can provide an iodine distribution map image. These maps represent the cartography of iodine content in the lung parenchyma at the time point of the first pass of contrast media in each examination [4]. Recent softwares (Syngo MultiModality; Siemens Healthcare, Erlagen, Germany) have been suggested for quantification of lung perfusion blood volume (PBV), allowing objective evaluation [3] [4]. Few reports have investigated pulmonary perfusion correlating with pulmonary artery pressure (PAP) in patients with chronic pulmonary diseases.

The purpose of this study was to evaluate the automated quantification of the lung PBV, and its correlation with the estimated systolic pulmonary artery pressure (sPAP). We also evaluated the relationship between automated quantification of the lung PBV and underlying diseases in the high PAP group.

\section{Materials and Methods}

\subsection{Patient Population}

This retrospective study was approved by the Institutional Review Board. Patients with acute pulmonary thromboembolism were excluded based on CT findings in this study $(n=34)$. We selected patients who underwent both echocardiography and DECT angiography between May 2009 and December 2013 at our hospital. Two hundred and seventy-four patients (114 men) who underwent echocardiography within two weeks also underwent DECT angiography DECT was performed for suspected pulmonary hypertension (PH). Of these, 63 patients (27 men, 36 women; mean age, $63.9 \pm 15.5$ years) were defined as a high sPAP group, with an estimated systolic pulmonary arterial pressure (sPAP) of $\geq 40 \mathrm{mmHg}$ on echocardiography [5]-[7]. The remaining 211 (123 men, 88 women; mean age, $65.9 \pm 15$ years) patients were defined as low sPAP group (Table 1) [5]-[7]. In subjects with a high sPAP, the underlying diseases were determined based on medical records and CT findings by a radiologist (over ten years of experience) as follows: pulmonary artery stenosis $(\mathrm{n}=12)$ [idiopathic $\mathrm{PH}(\mathrm{n}=10)$ and congenital shunt disease $(n=2)$ ], left heart failure $(n=11)$ [mitral valve stenosis $(n=7)$, aortic valve stenosis $(n=3)$, and/or mitral valve regurgitation $(n=3)$ ], lung disease $(n=15)$, and chronic thromboembolism $(n=$ 25), using the Dana Point classification system [7]-[9]. Lung diseases included chronic obstructive disease $(C O P D)(n=10)$ and interstitial pneumonia $(n=15)$ [idiopathic interstitial pneumonia $(n=10)$ and due to other collagen diseases $(n=3)$ ].

In subjects with a low SPAP, the underlying diseases were also determined based on medical records and CT findings as follows: no disease $(n=167)$, pulmonary artery stenosis $(n=11)$ [idiopathic $\mathrm{PH}(\mathrm{n}=8)$ and congenital shunt disease $(n=3)$, lung disease $(n=18)$, and chronic thromboembolism $(n=15)$. Lung diseases included chronic obstructive disease (COPD) $(n=10)$ and interstitial pneumonia $(n=8)$ [idiopathic interstitial pneumonia $(\mathrm{n}=5)$ and due to other collagen diseases $(\mathrm{n}=3)]$.

Table 1. Patient Characteristics, lung PBV value, and sPAP between high and low sPAP groups.

\begin{tabular}{cccc} 
& High sPAP group $(\mathrm{n}=63)$ & Low sPAP group $(\mathrm{n}=211)$ & P Value \\
\hline Age (y.o.) & 63.9 & 65.9 & NS \\
Number of male & 27 & 123 & NS \\
Lung PBV value (HU) & $25.0 \pm 9.6$ & $29.0 \pm 9.3$ & 0.003 \\
sPAP (mmHg) & $56.5 \pm 9.1$ & $26.8 \pm 8.6$ & $<0.0001$ \\
\hline
\end{tabular}

Note: sPAP means systoric pulmonary artery pressure; HU means Hounsfiled units. 


\subsection{CT Acquisition Protocol}

In all patients, we used a dual-source CT scanner (Somatom Definition; Siemens Healthcare, Erlagen, Germany) in dual-energy mode. Firstly, precontrast CT of the chest was conducted.

High-concentration iodine-based contrast material (Omnipaque 350; Daiichi-Sankyo, Tokyo, Japan) was administered at a flow rate of $4.0 \mathrm{~mL} / \mathrm{s}$ followed by a $40-\mathrm{mL}$ saline chaser bolus at the same injection rate. The total volume of contrast material was adapted to the patient's body weight at $1.35 \mathrm{ml} / \mathrm{kg}$. In this study, CT scanning began at a fixed $14 \mathrm{~s}$ from the start of injection [3] [4]. To avoid streak artifacts due to highly concentrated contrast material in the subclavian vein or superior vena cava, scans were acquired in the caudocranial direction. Other scan parameters were as follows: tube voltage, 140 and $80 \mathrm{kVp}$ at 30 and 210 effective mAs; attenuation-based tube current modulation; rotation time, $0.5 \mathrm{~s}$; collimation, $14 \times 1.2 \mathrm{~mm}$; and pitch, 0.7 . T CT angiography (CTA) images were reconstructed with a specific medium-soft convolution kernel (D30) without edge modification at a $1.0-\mathrm{mm}$ slice thickness with a $1.0-\mathrm{mm}$ increment. The image reconstruction system generated three stacks of axial images: $140-\mathrm{kV}$ images, $80-\mathrm{kV}$ images, and weighted-average images that derived $60 \%$ of the image density from the $140-\mathrm{kV}$ images and $40 \%$ from the $80-\mathrm{kV}$ images to achieve an image quality and a signal-to-noise ratio similar to those of single-energy $120-\mathrm{kV}$ scans [3] [4].

\subsection{Echocardiography Procedure}

Doppler transthoracic echocardiography examination was performed by one cardiologist (over ten years of experience) using a GE Healthcare Vivid q (GE Medical Systems; Milwaakee, Wis). Estimated sPAP was obtained using the modified Bernoulli formula by measuring the peak tricuspid regurgitation velocity and estimation of central venous pressure from inferior vena cava compliance.

\subsection{Analysis of Dual-Energy Data}

On the basis of three-material decomposition of soft tissue, iodine, and air, iodine distribution maps of the lungs were made on a workstation (Syngo MultiModality; Siemens Healthcare) with specific dual-energy postprocessing software. Hounsfield units (HU) were automatically calculated in several patterns, including both lungs, and the left or right lungs [4]. We used the data from both lungs.

\subsection{Statistical Analysis}

All values are expressed as the means. Statistical analysis was performed using clinical and morphological variables with the paired t-test and Mann-Whitney's U-test for continuous variables. One-way analysis of variance (ANOVA) was used to test for significant differences in mean lung PBV values among the four causes of PH. If a significant difference was found, the Bonferroni-Dunn test was used for post-hoc analysis. Pearson correlation coefficients were used to examine correlations. Correlation coefficients of 0.4 - 1.0 were considered to indicate a correlation [10]. In all tests, $\mathrm{p}<0.05$ was considered significant (Statview J-5.0 for Windows; Spaces Concepts, Berkeley, Calif., USA).

\section{Results}

\subsection{Analysis of All Subjects}

Table 1 shows a summary of the results. For patient (whole lung)-based analysis, lung PBV values were $25.0 \pm$ 9.6 and $29.0 \pm 9.3$ in high and low sPAP groups, respectively. There was a significant difference between the two groups $(\mathrm{p}=0.003)$. However, no significant correlation between the lung PBV and PAP was observed $(\mathrm{R}=$ $-0.2177, \mathrm{p}=0.063)$ in all subjects $(\mathrm{n}=274)$ (Figure 1$)$.

\subsection{Analysis of Subjects with High SPAP}

In the high sPAP group with lung diseases $(\mathrm{n}=15)$, chronic thromboembolism $(\mathrm{n}=25)$, pulmonary artery stenosis $(n=12)$, and left heart failure $(n=11)$, lung PBV values were 18.6 $\pm 1.6,25.1 \pm 4.5,25.8 \pm 4.5$, and 32.7 $\pm 9.4 \mathrm{HU}$, respectively. One-way ANOVA was used to test for significant differences in mean lung PBV values among the four causes of PH. There were significant differences in quantification of the lung PBV among the 


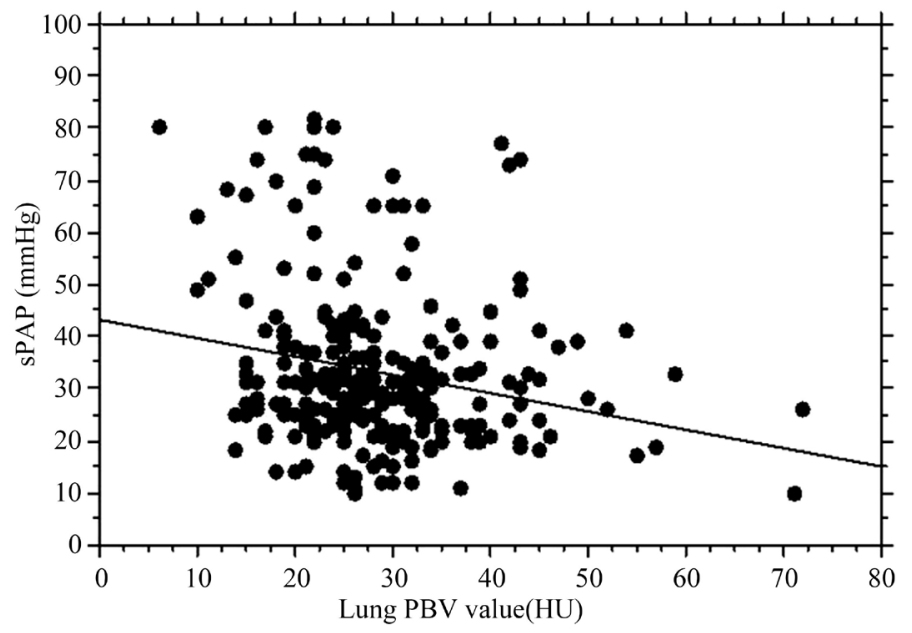

Figure 1. The relationship between the lung PBV and sPAP in all subjects $(\mathrm{n}=$ 274). No significant correlation between the lung PBV and PAP was observed $(\mathrm{R}=-0.2177, \mathrm{p}=0.0063)$.

underlying diseases $(\mathrm{p}<0.0001)$ (Figure 2).

Subjects with left heart failure had a significantly higher lung PBV than subjects with chronic thromboembolism $(p=0.0181)$ and pulmonary diseases $(p=0.0001)$. Subjects with pulmonary artery stenosis had a significantly higher lung PBV than subjects with pulmonary diseases $(\mathrm{p}=0.0349)$. Subjects with chronic thromboembolism had significant higher lung PBV value than those in subjects with pulmonary disease $(p=0.0246)$.

In the high sPAP group, no significant correlation between the lung PBV and sPAP was observed $(\mathrm{R}=-0.171$, $\mathrm{p}=0.1796)$ (Figure 3$)$.

In subjects with left heart failure, a positive correlation between the lung PBV and pulmonary artery pressure was observed $(\mathrm{R}=0.721, \mathrm{p}<0.0001$ ) (Figure 4(a)).

On the other hand, in subjects with lung disease $(\mathrm{R}=-0.487, \mathrm{p}<0.0001)$, chronic thromboembolism $(\mathrm{R}=$ $-0.415, \mathrm{P}=0.0391)$, and pulmonary artery stenosis $(\mathrm{R}=-0.385, \mathrm{P}=0.0047)$, a negative correlation was noted between the lung PBV and sPAP (Figures 4(b)-(d)).

We did not analysis the subjects with low sPAP group because many subjects had no the underlying diseases.

\section{Discussion}

PH is a progressive disease of the pulmonary arteries and is characterized by an abnormally elevated pressure in the pulmonary circulation, a result of extensive vascular proliferation and remodeling [8] [11] [12]. PH is defined as an abnormal elevation of pressure in pulmonary circulation, with a mean pulmonary arterial pressure higher than $25 \mathrm{mmHg}$ on catheterization of the right side of the heart, regardless of the underlying mechanism. $\mathrm{PH}$ is the hemodynamic consequence of vascular changes within the precapillary (arterial) or postcapillary (venous) pulmonary circulation.

Use of the term pulmonary arterial hypertension is restricted to those with a hemodynamic profile in which a high pulmonary pressure is a result of elevated precapillary and pulmonary resistance, a hemodynamic profile that is shared by groups 1, 3, 4, and 5 in the Dana Point classification system [5]-[8].

$\mathrm{PH}$ resulting from left heart disease (group 2) suggests an increase in the PAP due to the backward transmission of pressure elevation (postcapillary $\mathrm{PH}$ ).

In this study, the subjects were divided into high ( $\geq 40 \mathrm{mmHg}$ ) and low $(<40 \mathrm{mmHg})$ sPAP groups ( $\mathrm{n}=63$ and $\mathrm{n}=211$, respectively) based on the estimated sPAP using echocardiography because the prevalence of PH was defined as SPAP $\geq 40 \mathrm{mmHg}$ in some echocardiographic studies [5]-[7].

The correlation between the PAP estimated by echocardiography and a right heart catheterization study is good $(0.57$ - 0.85). However, when the PSP is determined by echocardiography, the hemodynamic value can be overestimated by $>10 \mathrm{mmHg}$ in up to $48 \%$ of patients, especially if the Doppler ultrasound recording is of poor quality. Furthermore, tricuspid regurgitation is found in approximately $80 \%$ of patients with a PSP $>35 \mathrm{mmHg}$ 


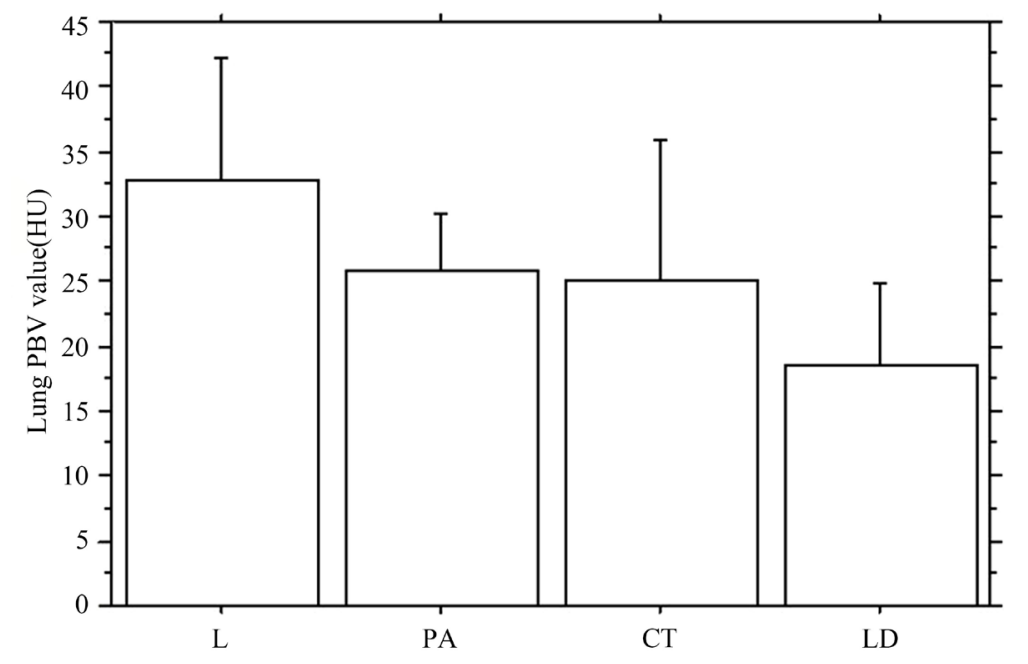

Figure 2. Individual lung PBV data in the high SPAP group with underlying diseases, such as lung diseases (LD), chronic thromboembolism (CT), pulmonary artery stenosis (PA), and left heart failure (L).

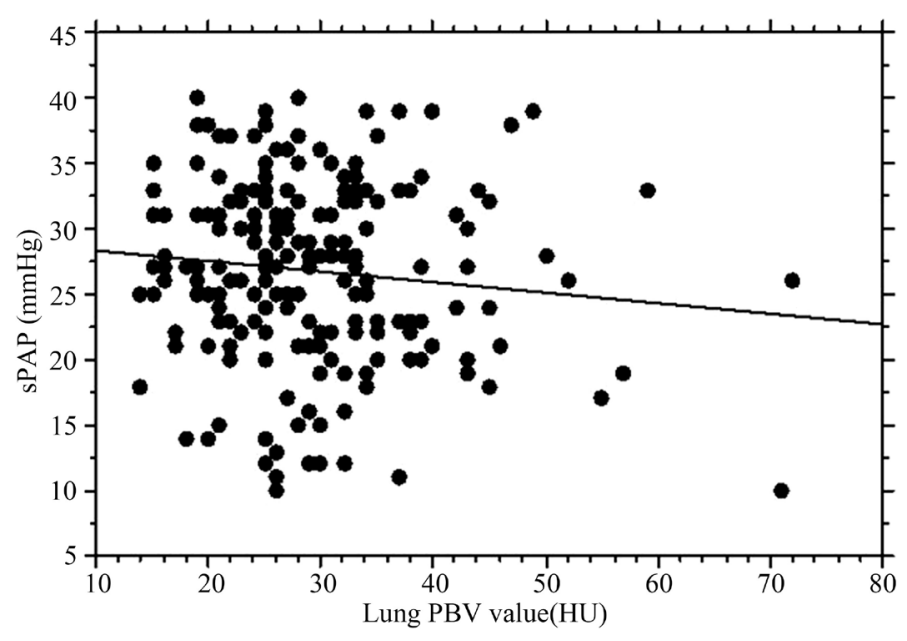

Figure 3. In the high sPAP group, no significant correlation between the lung PBV and sPAP was observed $(\mathrm{R}=-0.171, \mathrm{p}=0.1796)$.

and the ability to analyze flow varies according to the underlying disease [5]-[7]. Therefore, in this study, subjects with a high sPAP could not be diagnosed with PH because no underwent a catheterization study of the right side of the heart. This study used the estimated SPAP based on echocardiography, which is a limitation.

We found that there was a significant difference in the lung PBV between high and low sPAP groups. These results may indicate that pulmonary perfusion was reduced in subjects with a high sPAP, and the lung PBV may reflect a reduction in pulmonary perfusion.

There were also significant differences in lung PBV quantification among the underlying diseases in the high sPAP group. Subjects with left heart failure had a significantly higher lung PBV than subjects with other underlying diseases. In addition, a high positive correlation between the lung PBV and PAP was observed $(\mathrm{R}=0.721$, $\mathrm{p}<0.0001)$ in subjects with left heart failure. On the other hand, in subjects with lung disease, chronic thromboembolism, and pulmonary artery stenosis, a negative correlation between the lung PBV and pulmonary artery pressure was observed. There was a discrepancy between these results. Therefore, this study showed that there may be no significant correlation between the lung PBV and SPAP in the high sPAP group.

Pulmonary venous (postcapillary) hypertension is most frequently caused by left heart disease. Irrespective of the origin of left heart disease, the first event leading to $\mathrm{PH}$ is a passive backward transmission of filling pressures, 


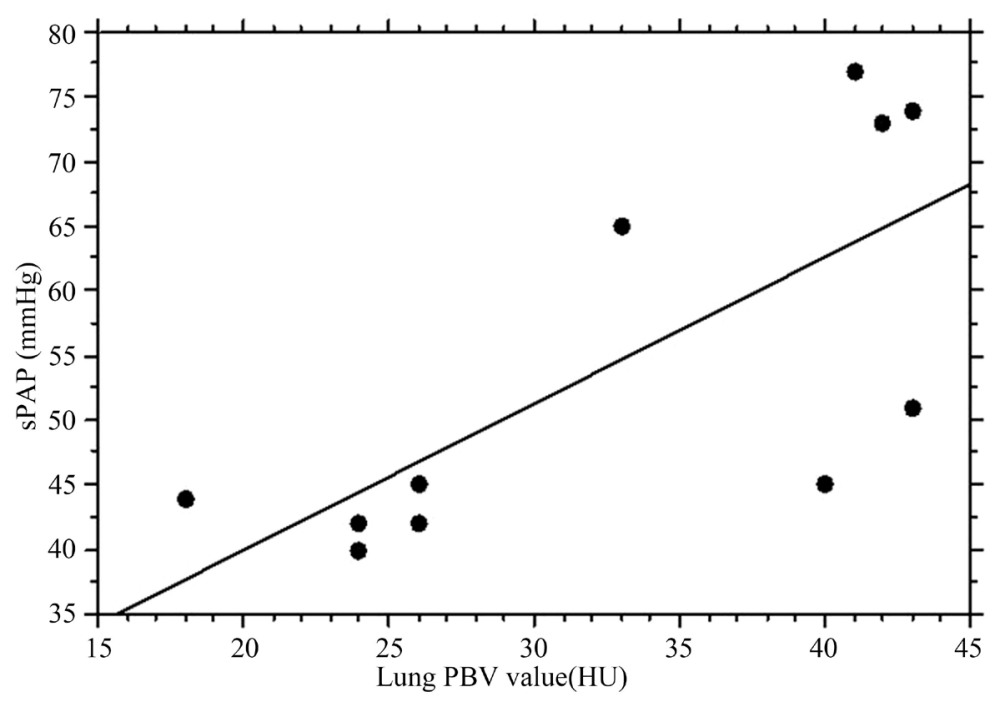

(a)

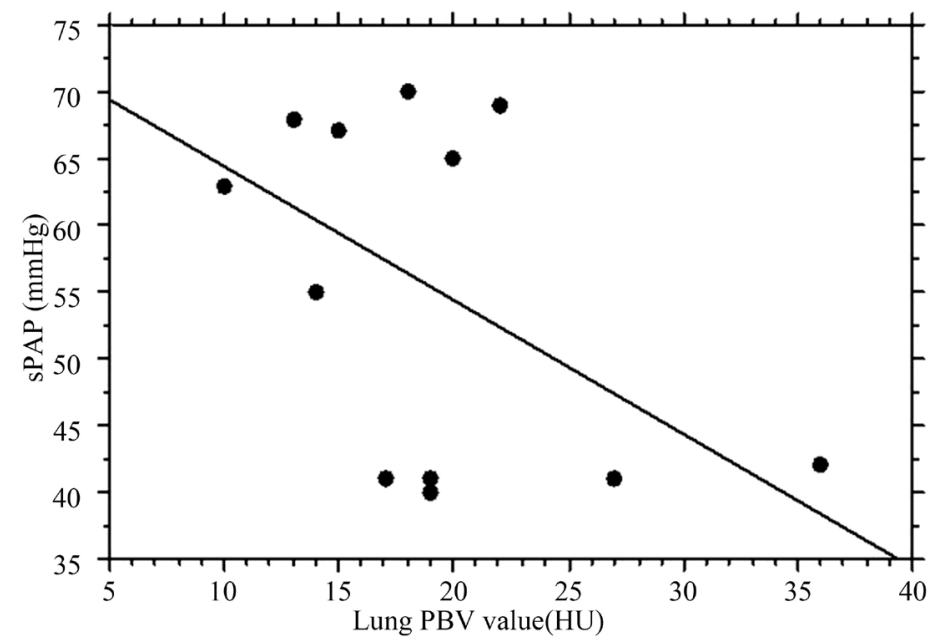

(b)

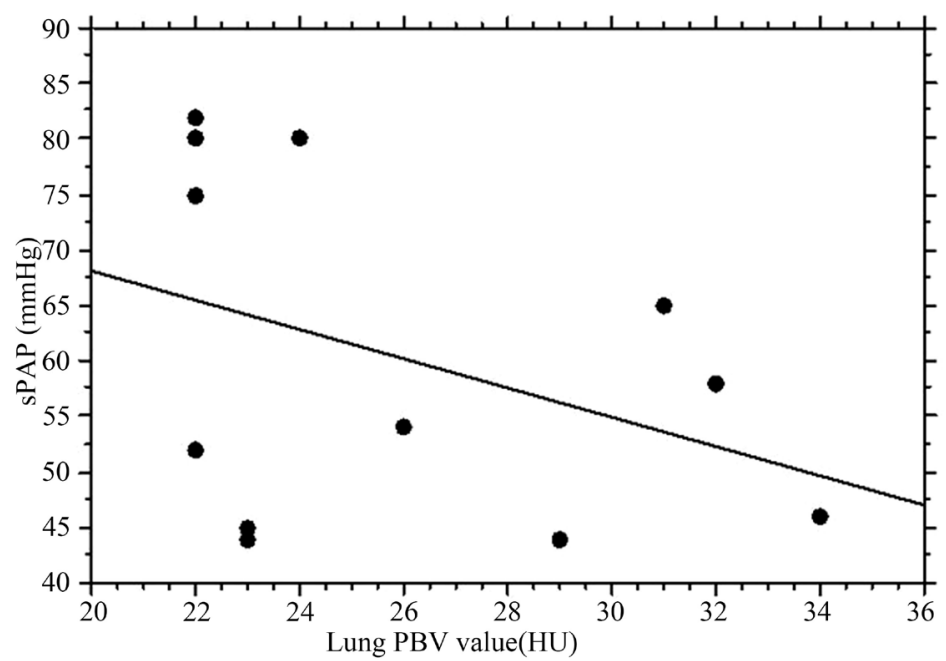

(c) 


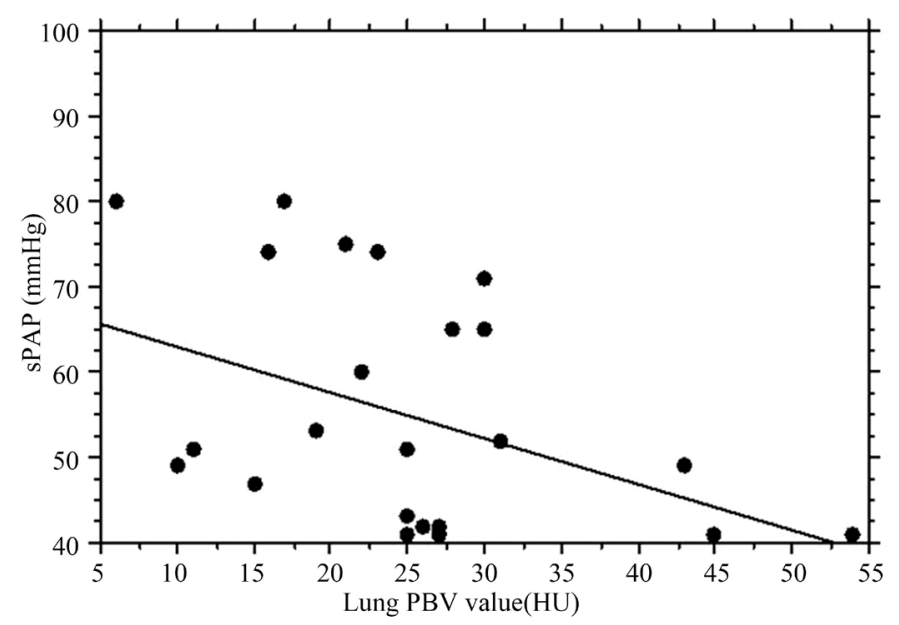

(d)

\begin{abstract}
Figure 4. (a) In subjects with left heart failure, a high positive correlation between the lung PBV and pulmonary artery pressure was observed $(\mathrm{R}=0.721, \mathrm{p}<0.0001)$; In subjects with lung disease $(\mathrm{R}=-0.487, \mathrm{p}<0.0001)$ (b), chronic thromboembolism ( $\mathrm{R}$ $=-0.415, \mathrm{P}=0.0391)(\mathrm{c})$, and pulmonary artery stenosis $(\mathrm{R}=-0.385, \mathrm{P}=0.0047)(\mathrm{d})$, a negative correlation between the lung PBV and SPAP was observed.
\end{abstract}

mainly driven by the left ventricular (LV) diastolic function. The resulting increase in the pulmonary artery wedge pressure (PAWP) may be enhanced by exercise-induced mitral regurgitation, together with a loss of left atrial compliance. The pulsatile load imposed by a chronically elevated PAWP might also play a role in the development of PH [13].

In subjects with left heart failure, the congestion of pulmonary blood occurs in postcapillary vessels of the PA (pulmonary vein). On the other hand, the congestion of pulmonary blood does not occur in postcapillary vessels of the PA in other groups with lung disease, chronic thromboembolism, or pulmonary artery stenosis. When left heart failure aggravates, the congestion of pulmonary blood in postcapillary vessels of the PA will worsen. Therefore, in subjects with left heart failure, the congestion of pulmonary blood in postcapillary vessels of the PA may cause a significantly high lung PBV, which may reflect the increase in the blood volume of both the pulmonary artery and vein. This theory supports the high positive correlation between the lung PBV and PAP observed $(\mathrm{R}=0.721, \mathrm{p}<0.0001)$ in subjects with left heart failure. However, further studies are needed to clarify this theory.

The other subgroups showed nocongestion of pulmonary blood in postcapillary vessels of the PA. In these subgroups, as narrowing of the pulmonary arteries progresses, the lung PBV will reduce. Therefore, in subjects with lung disease, chronic thromboembolism, and pulmonary artery stenosis, a negative correlation between the lung PBV and PAP was identified.

\title{
4.1. Clinical Implications
}

PH is a life-threatening condition characterized by an elevated PAP and pulmonary vascular resistance. However, no methods have been available for the quantification of pulmonary parenchyma perfusion itself. The lung PBV may be a useful tool for its quantification in PH. Additionally, lung PBV, CT, and CTA images can be obtained at the same time. Therefore, other serious causes of PH, including pulmonary embolism, can be excluded at the same time. As a result, lung PBV images may reduce the radiation exposure of patients.

In this study, there were significant differences in quantification of the lung PBV among the underlying diseases in the high PAP group. Especially, subjects with left heart failure had a significantly higher lung PBV than subjects with other diseases. These results suggest that the lung PVB can be used to estimate the etiology of PH. Additionally, this method, with its automatic quantification, is rapid and simple to use.

\subsection{Limitations}

There were several limitations of this study. Firstly, it included a limited number of patients, and all were diagnosed 
based on echocardiography. Therefore, the sPAP was not proved on right heart catheterization. Further studies are needed.

The second limitation was that coverage of the whole lung volume within this reduced FOV $(26 \mathrm{~cm})$ is not feasible. In these cases ( 81 of 253 cases), peripheral defects might be overlooked.

As the third limitation, CT scanning began at a fixed $14 \mathrm{~s}$ from the start of injection based on previous reports [3] [4]. This might underestimate the PBV in patients with suspected PH where the mean pulmonary transit time may be reduced.

\section{Conclusion}

Automated quantification of the lung PBV may estimate the high sPAP. The lung PBV may contribute to clarifying the etiology of a high PAP due to left heart failure.

\section{Conflict of Interest}

This work was supported by JSPS KAKENHI Grant Number 15K09894.

\section{References}

[1] Nef, H.M., Mollmann, H., Hamm, C., et al. (2010) Pulmonary Hypertension: Updated Classification and Management of Pulmonary Hypertension. Heart, 96, 552-559. http://dx.doi.org/10.1136/hrt.2008.156299

[2] Galie, N., Hoeper, M.M., Humbert, M., et al. (2009) Guidelines for the Diagnosis and Treatment of Pulmonary Hypertension: The Task Force for the Diagnosis and Treatment of Pulmonary Hypertension of the European Society of Cardiology (ESC) and the European Respiratory Society (ERS), Endorsed by the International Society of Heart and Lung Transplantation (ISHLT). European Heart Journal, 30, 2493-2537. http://dx.doi.org/10.1093/eurheartj/ehp297

[3] Sueyoshi, E., Tsutsui, S., Hayashida, T., et al. (2011) Quantification of Lung Perfusion Blood Volume (Lung PBV) by Dual-Energy CT in Patients with and without Pulmonary Embolism: Preliminary Results. European Journal of Radiology, 80, e505-e509. http://dx.doi.org/10.1016/j.ejrad.2010.10.011

[4] Nagayama, H., Sueyoshi, E., Hayashida, T., et al. (2013) Quantification of Lung Perfusion Blood Volume (Lung PBV) by Dual-Energy CT in Pulmonary Embolism before and after Treatment: Preliminary Results. Clinical Imaging, 37, 493-497. http://dx.doi.org/10.1016/j.clinimag.2012.08.007

[5] Gabbay, E., Yeow, W. and Playford, D. (2007) Pulmonary Arterial Hypertension (PAH) Is an Uncommon Cause of Pulmonary Hypertension (PH) in an Unselected Population: The Armadale Echocardiography Study. American Journal of Respiratory and Critical Care Medicine, 175, A713.

[6] Escribano Subias, P., Barberà Mir, J.A. and Suberviola, V. (2010) Current Diagnostic and Prognostic Assessment of Pulmonary Hypertension. Revista Española de Cardiología, 63, 583-596.

[7] Badesch, D.B., Champion, H.C., Sanchez, M.A., et al. (2009) Diagnosis and Assessment of Pulmonary Arterial Hypertension. Journal of the American College of Cardiology, 54, S55-S66. http://dx.doi.org/10.1016/j.jacc.2009.04.011

[8] Peña, E., Dennie, C., Veinot, J., et al. (2012) Pulmonary Hypertension: How the Radiologist Can Help. RadioGraphics, 32, 9-32. http://dx.doi.org/10.1148/rg.321105232

[9] Simonneau, G., Robbins, I.M., Beghetti, M., et al. (2009) Updated Clinical Classification of Pulmonary Hypertension. Journal of the American College of Cardiology, 54, S43-S54. http://dx.doi.org/10.1016/j.jacc.2009.04.012

[10] Fleiss, J.L. (1981) Statistical Methods for Rates and Proportions. 2nd Edition, Wiley, New York, 14-15.

[11] Rubin, L.J. (1997) Primary Pulmonary Hypertension. The New England Journal of Medicine, 336, 111-117. http://dx.doi.org/10.1056/NEJM199701093360207

[12] Runo, J.R. and Loyd, J.E. (2003) Primary Pulmonary Hypertension. The Lancet, 361, 1533-1544. http://dx.doi.org/10.1016/S0140-6736(03)13167-4

[13] Vachiéry, J.L., Adir, Y., Barberà, J.A., et al. (2013) Pulmonary Hypertension Due to Left Heart Diseases. Journal of the American College of Cardiology, 62, D100-D108. http://dx.doi.org/10.1016/j.jacc.2013.10.033 